\title{
O Impacto Do Uso Das Tecnologias Da Informação E Da Comunicação No Autoconceito E Na Qualidade De Vida Da Pessoa Idosa
}

\author{
The Impact Of The Use Of Information And Communication \\ Technologies In Self Concept And The Quality Of Life Of Elderly Person
}

\author{
Sónia de Almeida Ferreira ${ }^{1}$, Ana Isabel Veloso², Óscar Mealha ${ }^{3}$
}

\begin{abstract}
1.Sónia de Almeida Ferreira - É doutorada em Informação e Comunicação em Plataformas Digitais e mestre em Comunicação Multimédia pela Universidade de Aveiro. Investigadora no Centro de Estudos em Educação, Tecnologias e Saúde e Professora Adjunta Convidada do Instituto Politécnico de Viseu. soniaaferreira@ua.pt

2.Ana Isabel Veloso - É doutorada em Ciências e Tecnologias da Comunicação da Universidade de Aveiro; é mestre em Engenharia Biomédica e licenciada em Engenharia Informática pela Universidade de Coimbra. Atualmente é docente no Departamento de Comunicação e Arte da Universidade de Aveiro. Coordenadora de vários projetos de pesquisa - SEDUCE; EYE ON GAMES; Interactive Magic Places, People and Practices. A sua área de pesquisa são na comunicação mediada tecnologicamente, nas narrativas e jogos interativos, na interação humanocomputador. É autora de inúmeras publicações nacionais e internacionais. Orienta anualmente diversos alunos de Mestrado e de Doutoramento. Organizou a Conferência Videojogos2009.aiv@ua.pt

3.Óscar Mealha - É doutorado em Engenharia Electrotécnica pela Universidade de Aveiro, em 1995, tem cerca de 20 anos de investigação na área de Interacção Humano-Computador, usabilidade e visualização de informação, e destes, cerca de 16 anos a trabalhar para a área das Ciências e Tecnologias da Informação e Comunicação. É professor associado com agregação no Departamento de Comunicação e Arte da Universidade de Aveiro.oem@ua.pt
\end{abstract}

Resumo

Este artigo apresenta um estudo exploratório sobre o impacto da utilização das Tecnologias da Informação e da Comunicação nas variáveis psicossociais, autoconceito e qualidade de vida, da pessoa idosa e a influência das variáveis sociodemográficas. O trabalho foi desenvolvido em parceria com duas Instituições Particulares de Solidariedade Social do concelho de Aveiro, Portugal. Os instrumentos utilizados foram o Inventário Clínico de Autoconceito (Vaz-Serra, 1986) e Questionário de Qualidade de Vida da Organização Mundial de Saúde (Vaz-Serra et al., 2006). O estudo envolveu a participação de 12 idosos distribuídos por duas condições experimentais: seis utilizaram as Tecnologias da Informação e da Comunicação e seis não sofreram qualquer intervenção. Foram realizados dois momentos de avaliação, antes e depois de 11 meses de intervenção. Os resultados obtidos revelam que houve influência positiva das Tecnologias da Informação e da Comunicação na qualidade de vida dos participantes mas que não se verificou melhoria do autoconceito. Conclui-se, desta forma, que é importante integrar atividades de utilização das Tecnologias da Informação e da Comunicação no quotidiano da pessoa idosa. 
Palavras-chave: Tecnologias da Informação e da Comunicação, Idoso, Autoconceito, Qualidade de Vida

\begin{abstract}
This article presents an exploratory study on the impact of the use of information and communication technologies in the psychosocial variables, self-concept and life quality of the elderly person and the influence of socio-demographic variables. The work was developed in partnership with two private institutions of Social solidarity in the municipality of Aveiro, Portugal. The instruments used were the Clinical Inventory of Self-concept (Vaz-Serra, 1986) and quality of life questionnaire of the World Health Organization (Vaz-Sierra et al., 2006). The study involved the participation of 12 senior citizens spread over two experimental conditions: six used information and communication technologies and six didn't suffer any intervention. Two evaluation moments were performed before and after 11 months of intervention.

The results obtained reveal that there was positive influence of information and communication technologies on the quality of life of the participants but that there has been improvement of the self-concept. It is concluded, therefore, that it is important to integrate activities of use of information and communication technologies in everyday life of the elderly person.
\end{abstract}

Keywords: information and communication technologies, elderly, Self-concept, quality of life

57 FERREIRA, S.A.; VELOSO, A.I.; MEALHA, O.: O Impacto Do Uso Das Tecnologias Da Informação E Da Comunicação No Autoconceito E Na Qualidade De Vida Da Pessoa Idosa 


\section{Introdução}

O processo de envelhecimento é inerente à natureza de qualquer ser. Nos seres humanos, a passagem para a velhice não se limita a um conjunto de transformações biológicas. Existe um conjunto de mudanças que variam de acordo com o desenvolvimento psicossocial do indivíduo (Verona, et al., 2006). Para as teorias do envelhecimento bem sucedido (Ferreira \& Barham, 2011) e segundo a Organização Mundial de Saúde (WHO, 2002), conseguir que esse processo tenha êxito, ou seja, com autonomia física, psicológica e social, depende da capacidade do idoso de se envolver ativamente na proteção do seu bem-estar através da diversificação das atividades pessoais, do não cumprimento rígido de rotinas e da adesão a novas atividades mentais e físicas. O segredo está em implementar um estilo de vida que mantenha o corpo e a mente saudáveis e "aumentar a variedade de categorias de atividades ou evitar a sua perda" (Ferreira \& Barham, 2011: 581). A acompanhar a tendência demográfica de envelhecimento populacional está a velocidade de introdução das tecnologias em diversos espaços da nossa sociedade revelando-se fulcral colmatar o distanciamento que existe entre os idosos e as Tecnologias da Informação e da Comunicação (TIC).
Assim, justifica-se a realização de políticas e estudos que contribuam para a melhoria da qualidade de vida $(\mathrm{QV})$ na terceira idade e para a dissipar de preconceitos face aos idosos, não só pela valorização da dignidade da pessoa idosa, exigência estabelecida no segundo encontro da Assembleia Mundial do Envelhecimento das Nações Unidas (Pires, 2008) e pela União Europeia (EU, 2010), como pela satisfação de todos nós hoje por acreditarmos que amanhã seremos idosos.

O estudo coordenado por Espanha (2011), sobre o modo como a população portuguesa se relaciona com a Internet, mostra que existe claramente na nossa sociedade um fosso entre os mais novos e os mais velhos. Neste estudo, o perfil infoexclusão é constituído pelos indivíduos mais velhos, reformados, sem qualquer nível de escolaridade, de menores rendimentos e sem contacto com a Internet. Este é o grupo de pessoas que, diretamente, está relacionado com o nosso estudo.

Daqui por cerca de 20 anos, teremos os indivíduos que a pesquisa de Espanha (2011) identifica com o perfil não relação com a Internet. Este é constituído, na sua maioria, por pessoas com idades entre os 45 aos 64 anos, com um nível de escolaridade até ao segundo ciclo do ensino básico (seis anos de escolaridade) e aponta para situações em que a existência da Internet apenas responde à 
necessidade de utilização por parte de outros elementos do agregado familiar.

\section{Enquadramento}

\subsection{O Envelhecimento E A Relação Com As Tecnologias Da Informação E Da Comunicação}

Vários estudos revelam os benefícios da comunicação e partilha de informação mediada tecnologicamente nos idosos: melhoria geral do estado mental, reforço do autoconceito (AC) (Pires, 2008), da autorrealização e da autoestima (Sales, Guarezzi \& Filho, 2006); o aumento da QV (Leung \& Lee, 2005; Kiel, 2005); o bem-estar do idoso, tanto pelo perfil informativo e lúdico quanto pela possibilidade de integração no processo de aprendizagem (Miranda \& Faria, 2009); a melhoria das funções cognitivas e da depressão e o aumento do funcionamento diário (Whyte \& Marlow, 1999); a diminuição do sentimento de solidão (White, et al., 2002); a diminuição da perceção de stress (White \& Weatheral, 2000); e a nível do apoio social (Miranda \& Faria, 2009; Xie, 2008; White \& Weatheral, 2000). Contudo, o computador ao mesmo tempo em que contribui para melhoria da QV das pessoas também exclui quem não está interessado (Vianna, Bacha \& Santos, 2007). Purdie \& Boulton-Lewis (2003) verificaram que quando se confrontam os sujeitos com outras áreas de necessidades, como a saúde e o transporte, a necessidade de aprendizagem tecnológica perde destaque (Pires, 2008).

É inquestionável o papel que as Tecnologias da Informação e da Comunicação (TIC) possuem na sociedade moderna, porém, integrar as TIC no quotidiano da pessoa idosa é um desafio, atendendo a que este o grupo etário é o que menos utiliza as tecnologias computacionais. Czaja (2003) faz referência à pouca autoconfiança da população idosa quanto às suas capacidades para utilizar estas tecnologias, bem como, à ansiedade de execução das ações nas TIC. Proveniente de uma geração que sempre deteve o poder, o cidadão idoso passou a conviver com uma tecnologia que não faz diferença à sua vida, acabando por se afastar da tecnologia por motivos próprios de repúdio à inovação ou pelo entendimento das gerações mais novas que o caracteriza como um sujeito que não possui conhecimento e habilidade para a usar. Por sua vez, o que o idoso procura não é conhecer computadores e dominar a sua lógica, mas a apropriação, a inclusão como parte ativa e motivada por fazer parte da sociedade (Pasqualotti, 2008).

Paralelamente a estes estudos, dados avançados pelo INE (2009) indicam que 4,4\% dos indivíduos dos 65 a 74 anos utilizavam o 
computador, face a 42,5\% da população total (dos 16 aos 74 anos). Em 2002 essa percentagem era de $1,8 \%$.

Esta estatística corrobora a ideia de que a população idosa está mais recetiva às TIC e contrapõe o estereótipo de que os idosos são resistentes à sua utilização, questão que tem vindo a definhar desde os anos 80, com estudos como o de McMellon \& Schiffman (2002). Outras investigações evidenciam que, para além do interesse demonstrado na utilização das TIC, a pessoa idosa revela mesmo a aptidão no uso, embora se verifique que o género masculino as utiliza durante períodos de tempo mais longos (Sherer, 1997) e que precisam de mais tempo de treino e assistência durante a utilização do que outras camadas etárias (Charness, et al., 2001). Perante esta realidade, afirma-se a necessidade de investigar o impacto da utilização das TIC nas variáveis QV e AC da pessoa idosa.

\subsection{Autoconceito e Qualidade de Vida}

Vaz-Serra (1986) expõe que o Autoconceito (AC) é um construto psicológico que possibilita ter a noção da identidade da pessoa e da sua coerência e consistência. Acrescenta que é um construto teórico que nos esclarece sobre a forma como um indivíduo interage com os outros e lida com áreas respeitantes às suas necessidades e motivações, que nos leva a perceber aspetos do autocontrolo, porque algumas emoções surgem em contextos específicos ou porque uma pessoa inibe ou desenvolve determinado comportamento e permite-nos compreender a continuidade e a coerência do comportamento humano ao longo do tempo.

De acordo com Pires (2008) o AC pode ser influenciado por diversas variáveis. Têm sido feitos estudos sobre a influência da idade, da raça, do género e de várias situações indutoras de stress (como as doenças), entre outras variáveis. Relativamente às situações indutoras de stress, os preditores mais fortes do AC são as perturbações que têm uma componente psicológica mais clara, como as perturbações mentais (Proud \& Proud, 1996).

Segundo Cárdenas (2007) o AC não é inato, as pessoas não nascem já com um conceito de si próprias. O AC é construído durante todas as etapas do desenvolvimento humano. Recebe influência das pessoas significativas do ambiente familiar e social e configurar-se na dinâmica das suas experiências de sucesso ou fracasso.

Alguns estudos têm mesmo verificado que o efeito da idade no AC não é linear, isto é, declina na pré-adolescência e aumenta durante o fim da adolescência e o início da idade adulta (Crain, 1996). Whitbourne \& Sneed (2002) concordam e referem www.fics.edu.br 60 
que os idosos mantêm uma autoestima elevada e sentimentos positivos acerca do seu AC, apesar do confronto diário com avaliações negativas por parte da sociedade em que vivem. Contudo, esta não é a tendência esperada e Schaie \& Willis (2002) afirmam que o simples facto de envelhecer pode ter um impacto negativo no AC das pessoas, especialmente daquelas que têm necessidade de se ver sempre como pessoas com jovialidade e vitalidade.

\section{Cárdenas (2007) refere que o} comportamento da pessoa idosa depende não só da sua capacidade ou condição psicofísica como também da avaliação que faz de si. O sujeito que envelhece tende a viver em ambientes relativamente estáveis que permitem desempenhar atividades quotidianas com êxito. O surgimento das chamadas crises de identidade, decorrentes de doenças físicas incapacitantes, perdas de entes queridos e a possibilidade de perder a independência e o controle sobre sua própria vida, podem afetar de forma significativa o AC (Cárdenas et al., 2007).

Em Portugal, os estudos sobre o AC também demonstram uma tendência de manutenção de um AC elevado na velhice, como se pode confirmar no estudo de aferição do Inventário Clínico de Autoconceito de Vaz-Serra (1986). Segundo o autor os resultados obtidos revelaram que não existe qualquer diferença significativa entre o AC dos jovens adultos e o das pessoas com mais de 60 anos, apesar de estes últimos apresentarem a média de AC mais elevada.

A expressão Qualidade de Vida (QV) tem vindo a ser cada vez mais utilizada, historicamente, desde a década de 60 do século XX. Contudo, enquanto conceito científico revela-se ambíguo, a não ser que seja objeto de uma definição precisa (Vaz-Serra, et al., 2006). Fleck (2000) refere que a sua origem tem raízes no contexto político, apontando como referência o discurso do Presidente americano Lyndon Johnson que, em 1964, referiu que o progresso social deve ser medido através da QV proporcionada às pessoas e não pelo balanço dos bancos. Em 1948 a Organização Mundial de Saúde (OMS) redefiniu o conceito de saúde, caracterizando-se de forma mais abrangente, referindo-se a um estado de completo bem-estar físico, mental e social.

O interesse pela pesquisa na área da QV tem vindo a aumentar. Em 1969 apenas encontrava disponível uma única referência à QV na base de dados da Medline enquanto que, em 1995, encontrava 2424 referências (Pires, 2008). Apesar desse aumento de estudos científicos, verifica-se que ainda existe fraco consenso na definição deste conceito. 
O grupo de QV da divisão de Saúde Mental da Organização Mundial da Saúde apresenta um conceito que gera concordância entre as diversas definições. Definem a QV como a perceção que o indivíduo tem sobre a sua posição na vida, no contexto cultural e sistema de valores nos quais ele vive e em relação aos seus objetivos, expectativas, padrões e preocupações (Fleck, 2000).

O grupo criou então o instrumento de avaliação da QV, o WHOQOL-100, que avalia os seguintes seis domínios: o domínio físico, o domínio psicológico, o nível de independência, as relações sociais, o ambiente e os aspetos espirituais. A versão mais reduzida deste instrumento é o WHOQOLBref, que avalia quatro domínios: Físico, Psicológico, Relações Sociais e Ambiente.

$\mathrm{Na}$ tentativa de perceber a perspetiva dos idosos em relação à $\mathrm{QV}$, vários estudos têm sido feitos nesse sentido. Na perceção dos idosos a saúde interfere na longevidade, e acarreta melhor QV aos mesmos, sendo que os idosos que relataram excelente saúde aos 70 anos obtiveram uma expectativa de vida quatro anos superior em relação àqueles que consideraram sua saúde pobre (Chepp, 2006).

Gabriel \& Bowling $^{32}$ realizaram uma pesquisa da QV, em Inglaterra, com 999 pessoas com 65 ou mais anos a viver na comunidade. Dessa amostra, 80 participantes foram seguidamente entrevistados em maior profundidade durante um período de um a dois anos. Os temas associados à QV nas entrevistas de seguimento foram: possuir boas relações sociais, ajuda e suporte; viver numa casa e numa vizinhança que proporcionem prazer, segurança e acesso a transportes e serviços; participação em atividades de lazer e manutenção de um papel ativo na sociedade; possuir uma visão psicológica otimista e de aceitação das circunstâncias inalteráveis; possuir boa saúde e mobilidade; possuir dinheiro suficiente para satisfazer as necessidades básicas, para participar na sociedade, para desfrutar da vida e para conservar a sua independência e controlo sobre a vida.

Wilhelmson (et al., 2005) também estudaram a perspetiva dos idosos em relação à QV. Os resultados obtidos numa pergunta aberta sobre o que consideravam abranger a $\mathrm{QV}$ foram agrupados em oito categorias, por ordem decrescente de frequência de resposta: relações sociais; saúde; atividades; capacidade funcional; bem-estar; crenças e atitudes pessoais; o próprio lar e as finanças pessoais.

Os resultados destes dois estudos apresentam, assim, concordância quanto ao que os idosos consideram englobar a QV. Assim, e em virtude dos fatores associados empiricamente à $\mathrm{QV}$

www.fics.edu.br 62 
e aos fatores apontados pelos idosos, observamos que a definição apresentada pelo grupo de trabalho da OMS é bastante completa, uma vez que engloba os vários domínios que constituem o conceito.

De acordo com Haug e Folmar a mulher é quem apresenta uma QV inferior, em todos os indicadores. $\mathrm{O}$ género feminino possui desvantagem, principalmente quando mora sozinho. Segundo os autores as mulheres são mais propensas a sofrer com a falta de apoio, por viverem sozinhas, pelo decréscimo na saúde, o que indica uma QV inferior. Ocorre também relacionada com as aflições psicológicas, mostrando que as mulheres idosas apresentam maior tendência para estas situações (Chepp, 2006).

A perspetiva dos idosos portugueses é considerada bastante otimista. O estudo português de Simões (et al., 2001) sobre o bem-estar subjetivo dos idosos conclui que mesmo com as eventuais limitações das capacidades e perdas, os idosos sentem-se felizes. O estudo de Paúl também concluiu que os resultados são otimistas, em que a maioria dos idosos não avalia a sua QV negativamente. $\mathrm{O}$ estudo verificou que $3 \%$ dos idosos avaliam a QV como "Muito Boa”, 24\% como "Boa", 41\% como "Nem boa nem Má”, em oposição a 21\%, que a avaliam como "Má” e 11\% como "Muito Má". Apenas $27 \%$ se mostram efetivamente satisfeitos com a sua QV e a maioria (41\%) não se encontra nem satisfeita nem insatisfeita (Paúl, 2005).

Atendendo que a nossa pesquisa consiste na investigação das implicações na QV e no $\mathrm{AC}$ da pessoa idosa de um serviço tecnológico que é fulcral para a sua participação na sociedade digital, consideramos que este estudo se insere naquelas que devem ser as preocupações contemporâneas de todos perante os cidadãos em condições semelhantes. Em suma, um dos intuitos deste estudo é contribuir para reduzir as barreiras que se interpõem entre o cidadão e a informação, promovendo também o combate à discriminação tecnológica que os idosos têm sofrido, vistos como inábeis à utilização de plataformas digitais.

\section{Investigação Empírica}

\subsection{Procedimento metodológico}

Este estudo debruça-se sobre o impacto da utilização das TIC pelos idosos nas variáveis psicossociais, AC e QV. Além disso, procura-se 
averiguar se existe relação entre essas variáveis dependentes e as variáveis independentes associadas aos dados demográficos e ao contexto institucional, nomeadamente o género, o estado civil, o regime de frequência, tempo na IPSS, se recebe a visita de familiares e com que frequência, se recebe a visita de amigos e com que frequência, que atividades mais gosta de realizar, quem o orientou para frequentar a IPSS, se houve algum acontecimento que, no último ano, o tenha marcado significativamente e de que forma, a satisfação ao frequentar as sessões de informática (apenas para o Grupo A) e se acha que essas sessões influenciaram a sua vida (apenas para o Grupo A), quanto e como.

Assim, e de forma a dar corpo ao nosso estudo, definimos como hipótese de investigação: A utilização voluntária das TIC por um segmento de idosos institucionalizados influencia positivamente o seu AC e a sua QV.

Para a operacionalização do estudo estabeleceu-se uma parceria com duas Instituições Particulares de Solidariedade Social (IPSS) do concelho de Aveiro. Optou-se por realizar o estudo em Instituições Particulares de Solidariedade Social (IPSS) do concelho de Aveiro, Portugal (Litoral Centro), uma vez que os participantes idosos estariam, à priori, menos sujeitos a variáveis não controladas. A seleção das instituições foi feita depois do contacto formal, por carta, às onze instituições do concelho de Aveiro, cinco das quais mostraram interesse em fazer parte do estudo.
Posteriormente, fez-se uma visita guiada às IPSS a fim de conhecer as instalações, tomar conhecimento dos recursos informáticos disponíveis e saber quantos idosos estariam interessados em participar. Destas, selecionou-se duas instituições, que mostraram grande sensibilidade às necessidades de pesquisa na área da gerontotecnologia.

$\mathrm{Na}$ perspetiva de apresentar um contributo para o estudo das especificidades dos idosos, desenhou-se um estudo quase experimental, num contexto em que não foi possível a constituição de grupos aleatórios de participantes. Estabeleceu-se como critérios de seleção dos participantes: a idade superior a 65 anos, o estado cognitivo considerado normal, saber ler e escrever e participar voluntariamente no estudo. De referir que a constituição dos grupos participantes foi realizada com o total apoio das animadoras e assistentes socais de ambas as instituições.

Para a concretização do estudo criaram-se dois grupos de participantes: O grupo A, designado de grupo experimental, e o grupo B, constituindo o grupo de controlo passivo. O grupo A esteve envolvido em atividades de utilização das TIC, enquanto que o grupo $\mathrm{B}$ não esteve sujeito a qualquer atividade adicional às comuns do dia a dia que já realizavam. Além disso, para que o nosso estudo fosse exequível e de forma a eliminar possíveis influências provocadas pelo ambiente 
institucional, optou-se por definir uma amostra constituída por seis idosos, três da IPSS A e três da IPSS B.

As sessões frequentadas pelo Grupo A realizaram-se duas vezes por semana, com uma duração média de 90 minutos. Planeou-se um conjunto de atividades que pressupunham a utilização do Microsoft Office Word (escrita e formatação de textos), a exploração do Windows Live Hotmail, Messenger e do Google.

Para a avaliação do AC aplicou-se o Inventário Clínico de Autoconceito (Vaz-Serra, 1986). Trata-se de um constructo central na mediação do comportamento dos indivíduos. Quanto melhor o AC melhor um indivíduo funciona e melhor é o seu ajustamento. Da mesma forma, melhor são as suas expetativas perante a vida em geral, os amigos, os pares sociais, os familiares, o próprio, os subordinados e os superiores. O Inventário Clínico de Autoconceito é composto por 20 questões que constituem quatro fatores: o fator 1 é indicativo de aceitação/rejeição social, o fator 2 é indicativo de autoeficácia, o fator 3 de maturidade psicológica e o fator 4 de impulsividade/atividade. $\mathrm{Na}$ avaliação da QV dos idosos utilizou-se a Escala de Qualidade de Vida WHOQOL-Bref (WHOQOL Group, versão portuguesa: Vaz-Serra et al., 2006). É constituída por 26 questões que avaliam 4 domínios: físico, psicológico, relações sociais e ambiente.
As questões que integram o domínio físico estão relacionadas com dor e desconforto, energia e fadiga, sono e repouso, mobilidade, atividades da vida quotidiana, medicação e capacidade de trabalho. O domínio psicológico envolve questões sobre sentimentos positivos, pensar, aprender, memoria e concentração, autoestima, imagem corporal e aparência, sentimentos negativos e espiritualidade. O domínio das relações sociais incluí as relações sociais, a rede de suporte e a atividade sexual. O domínio do meio ambiente integra questões relacionadas com segurança física e proteção, ambiente no lar, recursos financeiros, cuidados de saúde e sociais, oportunidades de adquirir novas informações e habilidades, participação e oportunidades de recreação e lazer, ambiente físico e transporte. Aquando da investigação foi assinado um formulário de consentimento pelos participantes, não contemplando o parecer do comité de ética na Universidade de Aveiro, Portugal, criado posteriormente. O programa informático utilizado para a análise estatística dos dados é o programa SPSS 19 - Statistical Package for Social Sciences.

\subsection{Participantes}

No estudo participaram 12 pessoas idosas, seis do grupo experimental e seis do grupo de controlo passivo. No grupo A, a média de idade é de 80 anos $(\mathrm{SD}=5,15 ;$ máximo=90; mínimo=73), enquanto que no grupo B é de 82,33 anos ( $\mathrm{SD}=6,72$;

65 FERREIRA, S.A.; VELOSO, A.I.; MEALHA, O.: O Impacto Do Uso Das Tecnologias Da Informação E Da Comunicação No Autoconceito E Na Qualidade De Vida Da Pessoa Idosa 
máximo=92; mínimo=73). $\mathrm{O}$ número de participantes do género masculino e feminino é igual em ambos os grupos. A maioria dos participantes são viúvos, frequentaram quatro anos de escolaridade e desenvolveram uma atividade profissional enquanto trabalhadores não qualificados (trabalhadores na agricultura).

\section{Resultados}

A Tabela 1 apresenta os resultados do Autoconceito (AC) e da Qualidade de Vida (QV) do Grupo A e do Grupo B. De forma a averiguar a hipótese traçada, comparou-se os resultados obtidos com o Inventário Clínico do Auto-Conceito e a Escala de Qualidade de Vida da Organização Mundial de Saúde (Schaie \& Willis, 2002), do pré com o pós-teste, quer do Grupo A, quer do Grupo B. 
Tabela 1 - Resultados do Autoconceito (AC) e da Qualidade de vida (QV), dos grupos A e B

\begin{tabular}{|c|c|c|c|c|c|c|c|c|}
\hline \multirow{3}{*}{ Variáveis dependentes } & \multicolumn{4}{|c|}{ Grupo A } & \multicolumn{4}{|c|}{ Grupo B } \\
\hline & \multicolumn{2}{|c|}{ Pré teste } & \multicolumn{2}{|c|}{ Pós teste } & \multicolumn{2}{|c|}{ Pré teste } & \multicolumn{2}{|c|}{ Pós teste } \\
\hline & $M$ & $S D$ & $M$ & $S D$ & $M$ & $S D$ & $M$ & $S D$ \\
\hline Autoconceito: t(AC) & 65,8 & 4,9 & $71,2 *$ & 6,1 & 65,5 & 4,6 & 71,3 & 6,2 \\
\hline Aceitação/Rejeição social & 15,3 & 1,4 & 15,7 & 1,7 & 15,5 & 2,2 & 17,7 & 1,8 \\
\hline Auto eficácia & 19 & 1,5 & 21,3 & 2,2 & 17,5 & 1,8 & 18,7 & 2,5 \\
\hline Maturidade psicológica & 12 & 1,5 & 14,2 & 2,1 & 13,3 & 1,7 & 14,3 & 2,2 \\
\hline Impulsividade - Atividade & 10 & 1,3 & 14,2 & 2,1 & 13,3 & 1,7 & 14,3 & 2,2 \\
\hline Qualidade Vida: $\mathbf{t}(\mathbf{Q V})$ & 56,3 & 15,9 & $62,5^{*}$ & 7,1 & 54,2 & 10,4 & 50 & 9,0 \\
\hline Físico & 57,1 & 5,5 & 55,9 & 6,2 & 50 & 9,0 & 50,9 & 8,7 \\
\hline Psicológico & 61,8 & 7,9 & 54,2 & 5,3 & 68,8 & 5,4 & 62,5 & 7,9 \\
\hline Relações Sociais & 69,5 & 5,4 & 70,8 & 6,9 & 56 & 4,7 & 73,6 & 9,3 \\
\hline Ambiente & 59,9 & 6,3 & 64,1 & 7,1 & 50,5 & 5,3 & 56,8 & 7,9 \\
\hline
\end{tabular}

Os valores apresentados estão arredondados a uma casa decimal

$M$ - Média da subamostra $\mid S D$ - Desvio Padrão (Standard Deviation)

n - Número de idosos por subamostra|* - Diferenças amostrais com significância estatística

67 FERREIRA, S.A.; VELOSO, A.I.; MEALHA, O.: O Impacto Do Uso Das Tecnologias Da Informação E Da Comunicação No Autoconceito E Na Qualidade De Vida Da Pessoa Idosa 
Relativamente ao AC verificou-se que houve um aumento quer no Grupo A (pré teste o $\mathrm{t}(\mathrm{AC})=65,8$; pós teste o $\mathrm{t}(\mathrm{AC})=71,2)$, quer no Grupo B (pré teste o $\mathrm{t}(\mathrm{AC})=65,5$; pós teste o $\mathrm{t}(\mathrm{AC})=71,3)$.

Procurou-se averiguar quais os fatores do AC que apresentam as maiores subidas nestes grupos. Verificou-se que o aumento global do AC no Grupo B ficou a dever-se ao acréscimo de todos os fatores, porém o domínio de aceitação/rejeição social foi o que experimentou um aumento mais significativo. O crescimento do AC no Grupo A deve-se sobretudo ao fator da autoeficácia e da maturidade psicológica. Os fatores de aceitação/rejeição e de impulsividade - atividade também aumentaram mas não de forma significativa.

Os resultados da QV dos idosos do Grupo A mostram que houve um aumento de 6,25 pontos. No pré teste obteve-se 56,3 pontos enquanto que no pós teste obteve-se 62,5 pontos. No Grupo A o domínio que teve um aumento mais acentuado foi o ambiente. Os domínios físico e psicológico obtiveram reduções. Os valores obtidos na QV do Grupo B diminuíram. No pós teste o grupo obteve 54,2 valores e no pós teste 50 valores. O domínio que mais contribuí para este decréscimo foi o psicológico. Contudo, os domínios físico e das relações sociais aumentaram.

Considera-se, ainda, frutuoso determinar se existe relação com outras variáveis independentes. Embora se tenham obtido diferenças nos resultados amostrais, importa referir que, estatisticamente, apenas se encontraram diferenças significativas (significância de $5 \%)$ no AC $\quad(t=4,53$ $p=0,00<0,05)$ e na QV $(t=4,58 p=0,00<0,05)$ do GA. Apresentam-se apenas os resultados relativos às variáveis independentes género e instituição no momento de pós teste do Grupo A já que as restantes não possuem significado estatístico $(p<0,05)$.

Relativamente à variável género, os resultados indicam que a pecepção sobre o AC é mais elevada para os participantes masculinos $(\mathrm{rS}=0,45<0,7)$. Analisando a variável instituição, verifica-se que a instituição IPSS A apresenta valores de AC superiores em relação à IPSS B $(\mathrm{rS}=0,43<0,7)$.

No final da pesquisa os sujeitos foram questionados acerca da ocorrência de algum acontecimento significativo durante o estudo, situação que pode justificar a diferença que se verifica relativamente ao género e pode ser explicada em virtude de acontecimentos de natureza pessoal na vida de dois elementos do género feminino que compõem este Grupo A.

\section{Discussão Dos Resultados}

Os resultados obtidos neste estudo suportam a hipótese de que a utilização das Tecnologias da 
Informação e Comunicação (TIC) contribui para a melhoria da Qualidade de Vida (QV) da pessoa idosa em comparação com a não utilização das mesmas. Contudo o mesmo não acontece relativamente ao Autoconceito (AC).

Os resultados indicam que a Qualidade de Vida (QV) aumentou no grupo A, grupo envolvido com as TIC, e diminuiu no grupo B. No entanto o AC dos idosos aumentou quer no Grupo A quer no Grupo B, logo não poderemos relacionar com a utilização das TIC. Quando se tentou perceber quais dos fatores que compõem as variáveis $\mathrm{AC}$ e QV mais contribuíram para o acréscimo do AC, verificou-se que todos eles sofreram aumentos. O fator de AC que sofreu maior crescimento no grupo $\mathrm{B}$ foi o de "Aceitação/Rejeição social” e no grupo A foi o da "Autoeficácia".

Estes resultados obtidos relativamente ao AC são corroborados pelos estudos de VazSerra (1986), Crain (1996), Whitbourne e Sneed (2002) e Cárdenas (et al, 2007), que verificaram um AC elevado da pessoa idosa. Contudo, não se traduz nos estudos de Schaie e Willis (2002) quando afirmam que o simples facto de envelhecer tem impacto no AC das pessoas. Em relação à QV do grupo A fator que mais contribuiu para a subida verificada foi o "Ambiente" e aqueles que experimentaram decréscimos foram o "Psicológico" e o "Físico". No grupo B verificou-se um decréscimo considerável no fator "Psicológico" da QV e um crescimento mais vincado relativamente ao "Ambiente". Em relação à QV, que sofreu acréscimo, os resultados apresentados por Simões (et al., 2001) mostram que mesmo com as eventuais limitações físicas os idosos sentemse felizes, tal como se verificou neste estudo, houve diminuição do fator "Psicológico", no grupo A, mas mesmo assim a $\mathrm{QV}$ aumentou. Tentou-se perceber, então, se houve influência das variáveis independentes, género e instituição, nos dados obtidos. Não se contemplaram as variáveis independentes idade, estado civil, atividade profissional e regime de frequência da instituição uma vez que não se obtiveram valores passíveis de comparação.

De acordo com Haug \& Folmar o género feminino experimenta níveis baixos de $\mathrm{QV}$, e este estudo também revela isso. O género masculino obteve valores mais elevados ou iguais em relação ao género feminino, quer relativamente à $\mathrm{QV}$, quer ao $\mathrm{AC}$, à exceção do pós teste aplicado ao Grupo B, onde se verificou um acréscimo mais elevado de pré e pós teste no género feminino.

Os dados conseguidos relativamente à influência do contexto instituição mostraram que apenas podemos obter conclusões face à QV dos idosos. Os elementos da IPSS A conseguiram um aumento superior face aos indivíduos da IPSS B, em ambos os grupos. Estes resultados são apenas indicadores e não podem ser confrontados com outros estudos, 
uma vez que na revisão do estado da arte não se encontrou nenhuma pesquisa prévia feita com esta hipótese e abordagem metodológica.

\section{Conclusão}

Perante os resultados supra referidos, conclui-se ser pertinente e atual a concretização de contributos e iniciativas que fomentem a integração da pessoa idosa na sociedade cada vez mais tecnológica, objetivando a melhoria de variáveis psicossociais.

\section{Agradecimentos}

Agradece-se às Instituições Particulares de Solidariedade Social (IPSS) parceiras no projeto SEDUCE, Patronato de Nossa Senhora de Fátima de Vilar e ao Centro Paroquial de São Bernardo, toda a disponibilidade que demonstraram em acolher este projeto e em nos receber nas instituições permitindo uma verdadeira troca de saberes e valores.

Um agradecimento muito especial a todos os participantes que se envolveram no estudo, pela motivação e empenho que demonstraram, tornando tudo possível. Esta investigação do projeto SEDUCE foi financiada pela Fundação para a Ciência e Tecnologia e cofinanciado pelo Programa Operacional
Temático Factores de Competitividade (FCTPTDC/CCI/COM/111711/2009

COMPETE-FCOMP-01- 0124-FEDER014337).

\section{Referências Bibliográficas}

CÁRDENAS, C. J., et al. Espelho meu, existe alguém mais bonita (o) do que eu?: Um estudo do autoconceito com idosos. XIV Encontro Nacional da ABRAPSO. Rio de Janeiro: ABRAPSO. 2007.

CHARNESS, N., et al. Word processing trainning and retraining: effects of adult, experience and interface. Psychology and Aging. 16(1):110-27, 2001.

CHEPP, C. Estudo transversal da qualidade de vida através da Escala WHOQOL-Bref da população octogenária e nonagenária de Siderópolis. [dissertação]. Criciúma, Santa Catarina: Universidade do Extremo Sul Catarinense, 2006.

CRAIN, M. The influence of age, race and gender on child and adolescent selfconcept. In: Bracken BA, editor. Handbook of Selfconcept: developmental, social and clinical considerations. New York: John Wiley \& Sons; 395-420, 1996.

www.fics.edu.br 
CZAJA, S. J., et al. Factors Predicting the Use of Technology: Findings From the Center for Research and Education on Aging and Technology Enhancement (CREATE). Psychology and Aging. 21(2):333-52, 2006.

ESPANHA, R. A. Relação entre TIC, Utentes, Profissionais e Redes Tecnológicas de Gestão de Informação em Saúde. Lisboa: Centro de Investigação e Estudos em Sociologia, Instituto Universitário de Lisboa, 2011.

EU. Work session on demographic projections. In: Union E, editor. Lisboa: Eurostat European Commission, 2010.

FERREIRA, H. G., BARHAM, E.J. O Envolvimento de idosos em atividades prazerosas: Revisão da Literatura sobre Instrumentos de Aferição. Revista Brasileira de Geriatria e Gerontologia. 14(3): 579-90, 2011.

FLECK, M. P., et al. Aplicação da versão em português do instrumento abreviado de avaliação da qualidade de vida "WHOQOLbref". Revista de Saúde Pública. 34:178-83. 2000.
GABRIEL, Z., BOWLING, A. Quality of life from the perspectives of older people. Ageing \& Society. 24:675-91, 2004.

INE. Dia Internacional do Idoso: nos próximos vinte e cinco anos o número de idosos poderá mais do que duplicar o número de jovens. Lisboa, Portugal: Instituto Nacional de Estatística. 2007.

KIEL, J. M. The digital divide: Internet and e-mail use by the elderly. Med Inform Internet Med. 30(1):19-23, 2005.

LEUNG, L., LEE, P. S. N. Multiple determinants of life quality: the roles of internet activities, use of new media, social support, and leisure activities. Telemat Inf. 22(3):161-80, 2005

MCMELLON, C. A., SCHIFFMAN, L. G. Cybersenior Empowerment: How Some Older Individuals Are Taking Control of Their Lives. Journal of Applied Gerontology. 21(2):157-75, 2002.

MIRANDA, L., FARIAS, S. F. Contributions from the internet for elderly people: a review of the literature. Interface - Comunicação, Saúde, Educação. 13(29):383-94, 2009.

71 FERREIRA, S.A.; VELOSO, A.I.; MEALHA, O.: O Impacto Do Uso Das Tecnologias Da Informação E Da Comunicação No Autoconceito E Na Qualidade De Vida Da Pessoa Idosa 
PASQUALOTTTI, A. Comunicação, tecnologia e envelhecimento: significação da interação na era da informação. [tese]. Rio Grande do Sul: Universidade Federal Rio Grande do Sul., 2008.

PAÚL, C., et al. Satisfação e qualidade de vida em idosos portugueses. In: Paúl, C., Fonseca, A., editors. Envelhecer em Portugal: Psicologia, Saúde e Prestação de Cuidados. Lisboa: Climepsi; 77-95, 2005.

PFEIL, U., ZAPHIRIS, P., WILSON, S. Online social support for older people: characteristics and dynamics of social support. Workshop Enhancing interaction spaces by social media for the elderly; Vienna, 2009.

PIRES, A. T. Efeitos dos Videojogos nas Funções Cognitivas da Pessoa Idosa. Porto: Faculdade de Medicina do Porto, 2008.

PROUT, H. T., PROUT, S. M. Global selfconcept and its relationship to stressful life conditions. In: Bracken BA, editor. Handbook of Self-concept: developmental, social and clinical considerations. New York: John Wiley \& Sons; 259-86, 1996.
PURDIE, N. M., BOULTON-LEWIS, G.M. The Learning needs of older adults. Educational Gerontology. 29(2):129-49, 2003.

SALES, M. B., GUAREZZI, R. C., FILHO, F. Infocentro para terceira idade: relato de uma experiência por pares. Colabor@ - Revista Digital da CVA-RICESU. 4(13), 2006

SCHAIE, W., WILLIS, S. Adults Development and Aging. 5 ed. Riverin US, editor. NJ: Prentice Hall. 2002.

SHERER, M. Introducing computers to frail residents of homes for the aged. Educational Gerontology. 23:345-58, 1997.

SIMÕES, A., FERREIRA, A., LIMA, P. M., PINHEIRO, M., VIEIRA, C., MATOS, P. M., et al. O bem-estar subjectivo dos idosos: Factores sociodemográficos e de personalidade, Modelos e Práticas em Educação de Adultos. NAPFA. 301-20, 2001.

VAZ-SERRA, A. O Inventário Clínico de Auto-Conceito. Psiquiatria Clínica. 67-84. 1986.

VAZ-SERRA, A., et al. Estudos Psicométricos do Instrumento de Avaliação da Qualidade de Vida da Organização 
Mundial de Saúde. Psiquiatria Clínica. 27(1):41-9, 2006.

VERONA S., et al. Percepção do Idoso em relação à Internet. Temas em Psicologia. 14(2):189-97. 2006.

VIANNA, N., BACHA, M., SANTOS, J. Tecnologia da Informação e Terceira Idade: uma análise na ótica de estado de espírito com relação à atual. SEGeT - IV Simpósio de Excelência em Gestão e Tecnologia; 22-24 de outubro de 2007; Resende, Brasil. http://www.aedb.br/seget/artigos2007.php?pa g=34: Associação Educacional Dom Bosco AEDB. 2007.

WHO, World Health Organization. Active Ageing: A Policy Framework. Retrieved from http://www.who.int/ageing/publications/activ e_ageing/en/. 2002.

WHITE, H., et al. A randomized controlled trial of the psychosocial impact of providing internet training and access to older adults. Aging and Mental Health. 6(3):213-21, 2002.

WHITE, J. WEATHERALL, A. A grounded theory analysis of older adults and information technology. Educational Gerontology. 26(4):371-86, 2000.

WHITBOURNE, S., SNEED, J. The paradox of well-being, identity processes, and stereotype threat: Ageism and its potential relationships to the self in later life. In: Nelson T, editor. Ageism: Stereotyping and prejudice against older persons. Cambridge: MIT Press, 2002.

WILHELMSON, K., et al. Elderly peoples' perspective on quality of life. Ageing and Society. 25(4):585-600, 2005.

WHYTE, J., MARLOW, B. Beliefs and attitudes of older adults toward voluntary use of the internet: an exploratory investigation. OZCHI: Annual Conference of the Australian Computer-Human Interaction Special Interest Group; 1999; Wagga Waga, Austrália. 1999.

XIE, B. Multimodal Computer-Mediated Communication and Social Support among 